Editorial

\title{
COVID-19 Lockdown in Wuhan: Heart Rate Variability in Females and Males - A Pilot Study
}

Gerhard Litscher ${ }^{1,2,+,}{ }^{*}$, Tong $\mathrm{Li}^{2,+}$, Fengxia Liang ${ }^{2}$

1. Research Unit of Biomedical Engineering in Anesthesia and Intensive Care Medicine, Research Unit for Complementary and Integrative Laser Medicine, and Traditional Chinese Medicine (TCM) Research Center Graz, Medical University of Graz, 8036 Graz, Austria; E-Mail: gerhard.litscher@medunigraz.at

2. Hubei University of Chinese Medicine, Institute of Acupuncture \& Moxibustion, Wuhan, 430061 China; E-Mails: litong68686688@qq.com; fxliang5@hotmail.com

+ These authors contributed equally to this work.

* Correspondence: Gerhard Litscher; E-Mail: gerhard.litscher@medunigraz.at

Collection: Evidence-Based Integrative Medicine for Epidemics

OBM Integrative and Complementary Medicine

2021, volume 6 , issue 1

doi:10.21926/obm.icm.2101009
Received: March 14, 2021

Accepted: March 14, 2021

Published: March 15, 2021

\begin{abstract}
In a pioneer transcontinental pilot study conducted with 50 volunteers, it was revealed that women could have apparently overcome the world's toughest lockdown in terms of changes in the general state of health measured using the heart rate variability parameter better than men. The results of the present study obtained in Wuhan provide information on the initial trend in this regard.
\end{abstract}

\section{Keywords}

COVID-19; SARS-CoV-2 virus; heart rate; heart rate variability; gender; lockdown; Wuhan conditions of the Creative Commons by Attribution License, which permits unrestricted use, distribution, and reproduction in any medium or format, provided the original work is correctly cited. 


\section{Introduction}

Wuhan is probably the best place to explore the effects of an extremely strict lockdown on the health of individuals. The research center at the Medical University in Graz, which has been in intensive cooperation with the Hubei University for Chinese Medicine in Wuhan for several years, is the place where this investigation was taken up along with several other projects [1].

The global spread of COVID-19 has been keeping people in constant fear. The mental stress and anxiety that develops due to this fear may lead to mental illness, and in severe cases, it may also cause physical damage. Heart rate variability (HRV) reflects the functioning of the autonomic nervous system and, therefore, could serve as an indirect objective parameter representing good or bad health. A comparative analysis of relevant HRV indicators and mean heart rate (HR) in healthy people before and after the onset of the COVID-19 pandemic could, therefore, provide insights into whether the mental stress and fear caused by the COVID-19 pandemic and/or a strict lockdown exert an impact on the mental health of individuals.

\section{Heart Rate and Heart Rate Variability Prior to and After the Lockdown in Wuhan}

In order to analyze whether a correlation existed between the HRV of healthy people before and after the onset of the COVID-19 pandemic, a total of 50 healthy test subjects ( 27 women and 23 men; age range: $33.4 \pm 14.9$ years) were recruited from the Wuhan city in China. After the lifting of the world's first and toughest lockdown that was implemented on January 23, 2020, for a period of 76 days in Wuhan, 25 healthy subjects were recruited to be included in the COVID-19 group (August 2020 to January 2021). In addition, 25 healthy subjects were recruited retrospectively until before the beginning of the lockdown (2019), i.e., December 2019, to be included in the control group (clinical trial registration: ID NCT02924831). Patients from both groups were subjected to HRV tests in Wuhan using equipment from the Research Center for Traditional Chinese Medicine in the Medical University of Graz. The HRV analyses were also conducted in Graz. A paired sample test was performed for the COVID-19 group, and the control group using the SPSS24.0 software, and the level of significance was set at $P<0.05$. The possible changes in the HRV indices before and after the first lockdown during the COVID-19 crisis were compared to identify the possible changes in the subjects' mental stress and fear before and after the period. Initially, no difference was observed in the HRV-related indices (total HRV, SDNN, r-MSSD, VLF, LF, HF, and log LF/HF) between the COVID19 lockdown group and the control group ( $P>0.05$ for all). Overall, the psychological stress and fear due to COVID-19 lockdown exerted no medium-term impact on the HR and HRV of the healthy people in Wuhan. However, when a gender-specific analysis was conducted in extremely small groups within the pilot study, it was observed that men apparently had a reduced overall variability and a slightly increased mean HR compared to women after the lockdown (Figure 1 and Figure2). 

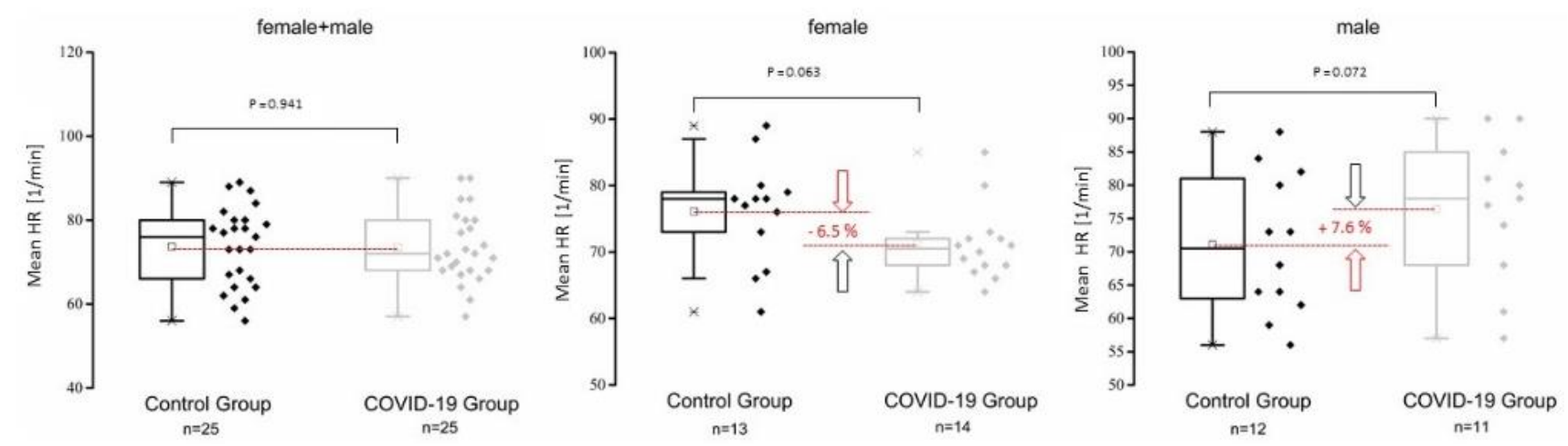

Figure 1 Box-plot representations of the mean heart rate (HR) of the females and males in the control group and COVID-19 group.
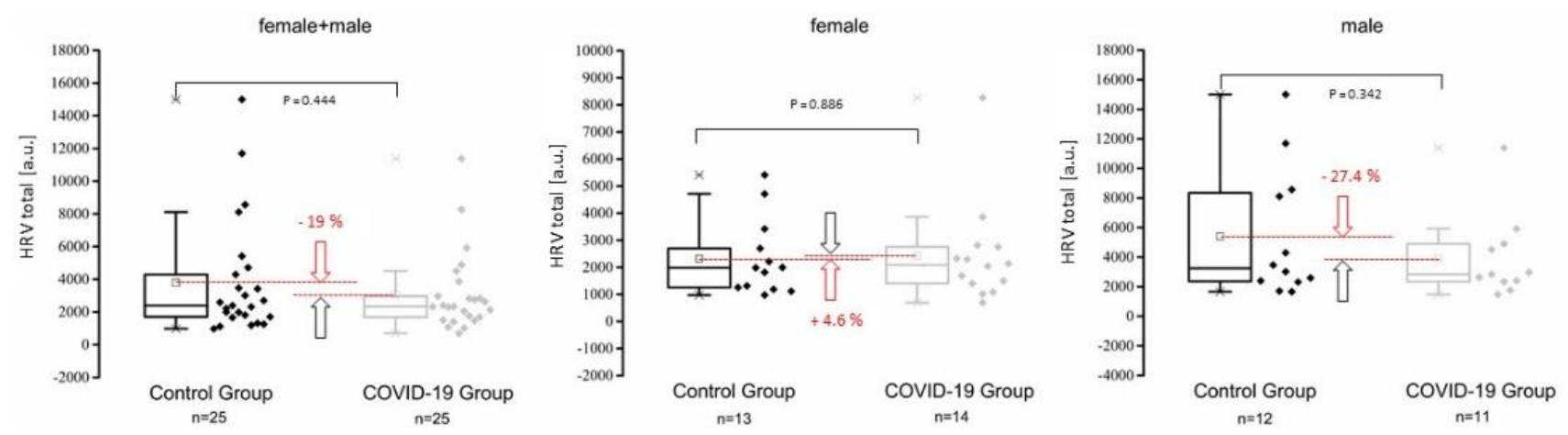

Figure 2 Box-plot representations of the total heart rate variability (HRV) of the females and males in the control group and COVID-19 group.

These findings of the joint research project will be presented at a public health conference in China in May 2021.

\section{Discussion}

HRV is a parameter that allows the quantification of the neuromodulation of the heart. In addition to being used in western research and practice, HRV is increasingly being used in evidencebased traditional medicine research. Currently, innovative research tools, including the most recent recording technology and the techniques of artificial intelligence for the acquisition and analysis of the HRV data, are also applied in the field of acupuncture. The different influences on the HRV are not yet completely understood, although it is known that there exist intra-individual and interindividual variances and that HRV is age-dependent. Moreover, the circadian alterations (sleepwake cycle), physical constitution, and mental and physical exertion are important influencing factors for HRV. HRV may also be caused by various diseases, including age-related and lifestyle diseases, such as diabetic neuropathy, kidney failure, essential hypertension, heart disease, coronary artery disease, and intracranial lesions. In the last few years, research related to HRV in the TCM Research Center in the Medical University of Graz has been focused on the latest innovative aspects of complementary medicine. Studies on various types of acupuncture techniques (manual needle acupuncture, laser acupuncture, and electroacupuncture) have been conducted on numerous patients, healthy volunteers, as well as animals, and the published findings are available 
for the scientific community worldwide [2]. In the present study, the electrocardiograms (ECG) were recorded in China, and the recorded data were immediately transferred to the Medical University of Graz via the internet (for example, acupuncture treatment). The analysis of the ECG was conducted in Graz. The acupuncturists in China (e.g., those in Wuhan, Harbin, Beijing) were immediately informed regarding the results of the analysis and, thus, the success of the therapy or the result of the investigation could be objectively proven. This type of teleacupuncture involves a multiple bridging function between Eastern and Western medicine, as well as between science and practice. This could contribute significantly to preventing redundant research studies and simplifying the diagnostic and therapeutic procedures, which would not only reduce the costs but would also save time. HRV could be considered a reliable indicator of the state of health of an individual and could be demonstrated to be applicable in certain special syndromes, such as fatigue and stress, to counteract a negative course with various prevention methods, including acupuncture. This has already been investigated in patients with burnout syndrome in transcontinental teleacupuncture studies between Wuhan and Graz [3].

In order to further progress the research during and after the COVID-19 pandemic, it would be interesting to quantitatively rate the well-being of individual groups, particularly among children and adolescents. In addition, quantifiable comparisons, with data from Europe [4] that include HRV as well as the stress and anxiety data, before, during, and after the lockdown, would be desirable.

\section{Conclusions}

Women apparently have better lockdown coping strategies along with overall health compared to men. The present transcontinental pilot study on HR and HRV conducted with 50 individuals from Wuhan provides information on the initial trend in this regard. Nonetheless, due to the limitations of this pilot study, further investigations are warranted.

\section{Acknowledgments}

The corresponding author of this editorial thanks Mr. Li Tong and Prof. Liang Fengxia for the joint development of the preliminary results and for the organizational and scientific support in Wuhan.

\section{Author Contributions}

All authors (G.L., T.L., and F.L.) made substantial contributions to conception and design, acquisition of data (T.L., F.L.), analysis (G.L., T.L.) and interpretation of data (G.L.,F.L.). G.L., T.L., and F.L. participate in drafting the article, revising it critically (G.L.) and all authors give final approval of the version to be submitted and the revised version.

\section{Funding}

The Eurasia-Pacific Uninet project EPU 36/2019 (project manager G. Litscher) was approved on April 1 $1^{\text {st }}, 2020$ in Austria. A short report on this project has been published in German language in 'Akupunktur und Aurikulomedizin' recently [5]. G.L. is also guest professor at the Hubei University of Chinese Medicine. 


\section{Competing Interests}

The authors have declared that no competing interests exist.

\section{References}

1. Li T, Liang FX, Litscher G. The general impact of mental stress and anxiety of COVID-19 on heart rate variability in healthy people in Wuhan. Proceedings of the 5 th International Conference on Public Health and Medical Science; 2021 May 29-31; Wuhan, Hubei Province. New York: Science Publishing Group.

2. Litscher G. Heart rate variability and acupuncture. Lengerich: Pabst Science Publishers; 2016.

3. Shu Q, Wang H, Litscher D, Wu S, Chen L, Gaischek I, et al. Acupuncture and moxibustion have different effects on fatigue by regulating the autonomic nervous system: A pilot controlled clinical trial. Sci Rep. 2016; 6: 37846.

4. Bourdillon N, Yazdani S, Schmitt L, Millet GP. Effects of COVID-19 lockdown on heart rate variability. PloS ONE. 2020; 15: e0242303.

5. Litscher $\mathrm{G}$ and the members of the Eurasia Pacific Uninet project team. COVID-19 lockdown in Wuhan and heart rate variability-a short report on a pilot study. Akupunktur Aurikulomedizin. 2021; 47: 40-42.

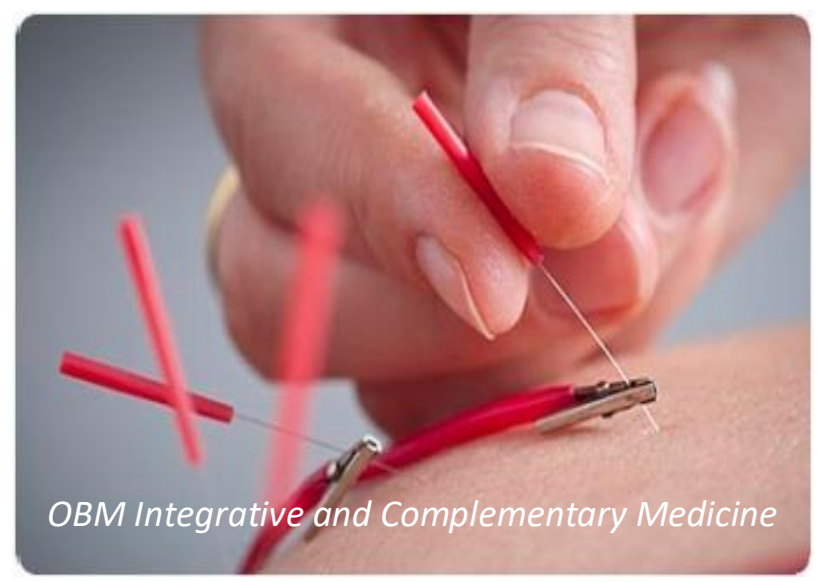

Enjoy OBM Integrative and Complementary Medicine by:

1. Submitting a manuscript

2. Joining in volunteer reviewer bank

3. Joining Editorial Board

4. Guest editing a special issue

For more details, please visit: http://www.lidsen.com/journals/icm 